\title{
Bargaining Solution-Based Resource Allocation Scheme for Cloud-Assisted Wireless Body Area Networks
}

\author{
Sungwook Kim (i) \\ Department of Computer Science, Sogang University, 35 Baekbeom-ro (Sinsu-dong), Mapo-gu, Seoul 121-742, Republic of Korea \\ Correspondence should be addressed to Sungwook Kim; swkim01@sogang.ac.kr
}

Received 12 June 2019; Revised 10 January 2020; Accepted 14 January 2020; Published 18 February 2020

Academic Editor: Miguel Garcia-Pineda

Copyright (c) 2020 Sungwook Kim. This is an open access article distributed under the Creative Commons Attribution License, which permits unrestricted use, distribution, and reproduction in any medium, provided the original work is properly cited.

\begin{abstract}
Wireless body area network (WBAN) is an emerging technology that has enormous potentials for continuous health monitoring of various diseases. For different medical and healthcare applications, the collected physiological data from multiple WBANs are further transmitted to the remote medical cloud servers. However, several technical issues and challenges are associated with the integration of WBANs and cloud computing services. In this study, we develop a new cloud-assisted WBAN control scheme to effectively use the limited system resource. By employing the main ideas of Generalized Gini and Choquet bargaining solutions, our approach unfolds into dual stages of bargaining processes while increasing the flexibility and adaptability. In particular, we consider the unique features of cloud-assisted WBAN applications and provide a generalized fair-efficient solution for the resource allocation problem. Numerical simulation results demonstrate that we can verify the superiority of our proposed scheme over the existing protocols. Lastly, major further challenges and future research directions about the cloud-assisted WBAN paradigm are summarized and discussed.
\end{abstract}

\section{Introduction}

With the rapid development of smart devices and Internetof-Things (IoT) technologies, wireless body area network (WBAN) can replace complex and offline-based healthcare process. To quickly provide real-time feedback to the patients, the patient's vital signals are continuously monitored. Some of the advantages of WBAN are (i) speeding up patients' recovery by online monitoring, (ii) decreasing healthcare expenditures, and (iii) increasing the quality of critical medical decision-making. Usually, the WBAN consists of several lightweight on-body or implanted smart edge devices to monitor physiological factors. For carrying out medical and healthcare diagnosis, the WBAN needs patients' current information, such as blood pressure, electrocardiogram signals, temperature, and heart rate, without interrupting patient's daily lifestyle $[1,2]$.

Increased number of WBANs in a specific local area degrades the performance of each WBAN in terms of endto-end packet delivery delay and network throughput. To make a fast diagnosis and provide a prompt medical response, the real-time online access to the vital factors of patients is necessary. In addition, during the WBAN operations, not only does collected data need to be processed on time, but also timely feedback is essential. Usually, WBAN applications consist of multiple computation tasks, which are becoming increasingly computation-intensive. However, due to its portable size, the computing capacity of each WBAN is limited. Therefore, it is difficult to achieve those goals by solely relying on a traditional WBAN's infrastructure. To deal with this technical problem, several methods have been developed and implemented; most of them have focused on investigating how to efficiently use the scarce WBAN resources [3-5].

In the last couple of years, cloud computing has been a nascent but fast-growing innovation that has attracted increasing attention from both the academia and the industry. As a new information technology paradigm, cloud computing is a novel service related to a distributed network technology. Through resource virtualization, the cloud computing provides computing resources and performs efficiently compute-intensive tasks. As a result, cloud 
computing can help overcome many of the inherent weaknesses in traditional WBANs services [4]. Fortunately, WBANs and cloud computing are not incompatible in nature; in fact, they can compensate each other's limitations. To achieve the required WBAN goals, a powerful approach is to offload some of their computation tasks to a remote cloud. It has inspired us to seek a joint solution by considering mutually dependent features between WBANs and cloud computing mechanism [5].

The integration of WBANs and cloud computing is expected to facilitate the development of cost-effective, scalable, and real-time online healthcare systems, which must be able to realize the data analysis of patients in different environments. As one of the most influential innovations, the cloud-assisted WBAN (CWBAN) systems are designed by integrating WBANs and cloud computing paradigm; they can migrate the computation tasks from each WBAN to the cloud server. Therefore, the CWBAN system can solve the most critical WBAN control issue. With sufficient computation capacity, the CWBAN system provides additional features such as the reduced deployment cost while sharing the critical information in real time to improve the decision-making process [5-7].

Based on the seamless integration of WBANs and cloud computing, the CWBAN system introduces several advantages. Most of all, a wider range of richer functional services can be provided with cloud computing capabilities; they meet richer application requirements. Therefore, performance efficiency is enhanced while reducing the resource constraints of edge devices in WBANs. In a real situation, there are some actual use cases for the CWBAN system. First, the CodeBlue project at Harvard University considers a hospital environment where multiple router nodes can be deployed on the wall. All nodes use the same ZigBee radio, and there is no centralized or distributed server or database for control and storage. Localization functionality is provided by MoteTrack with an accuracy of 1 meter where packet loss is limited to $40 \mathrm{~kb} / \mathrm{s}$ [8]. Second, Advanced Health and Disaster Aid Network is being developed at Johns Hopkins University for mass casualty incidents. Based on the communications between personal servers and the central server, a web portal is provided to multiple types of users, including emergency department personnel, incident commanders, and medical specialists [9]. Third, Wearable Health Monitoring System is being developed at the University of Alabama to target a larger-scale telemedicine system for ambulatory health status monitoring. The personal server, implemented on a personal digital assistant, cell phone, or personal computer, coordinates the data collection from sensor nodes using a time-division multiple access mechanism, provides an interface to users, and transfers data to a remote central server. Physicians can access data via the Internet, and alerts can be created by an agent running on the server [10].

Even though there have been some methods, the research on the CWBAN system is still in its infancy, and several technical issues and challenges are to be addressed in order to implement the practical CWBAN system. Therefore, finding a new control paradigm to solve these difficulties would be the top-priority task [6]. As a family of theories often subsumed under the umbrella term "rational choice theory," game theory is the science of strategy. It attempts to determine mathematically and logically the actions that game players should take to secure the best outcomes for themselves in a wide array of games. Therefore, game theory is really interested in the players' evaluations of this consequence, which are specified in each player's socalled payoff or utility function. Recently, game theory based resource allocation methods have been widely investigated in existing research work. They have been widely used to formulate different types of resource allocation problems in network management. The part of game theory that deals with situations in which players' choice of strategies cannot be enforced is called the theory of noncooperative games. In contrast, cooperative game theory allows for preplay agreements to be made binding through legally enforceable contracts [11].

According to the characteristics of CWBAN resource allocation problem, cooperative game models can fit more appropriately. In essence, such cooperative approaches are beneficially used for the communication and computation resource allocation problem in the CWBAN system. To this end, cooperative bargaining game models are chosen to design a novel resource allocation scheme for the CWBAN system. In this study, we adopt the ideas of Generalized Gini Bargaining Solution (GGBS) and Choquet Bargaining Solution (CBS) to effectively allocate the resources of CWBAN system. Based on the GGBS, the communication resource is allocated to each WBAN, and the computation resource of cloud system is distributed according to the CBS. These two solutions share a common principle of rationality; they are consistent with the maximization of some ordering of the utility space [12].

1.1. Contribution. To fulfill the promised advantages of CWBAN platforms, several technical issues and challenges should be addressed. In this study, our work addresses the communication and computation resource allocation problem in the CWBAN system. To model the interaction between each WBAN and cloud server, we design a new dual-phase cooperative game process. Using two different bargaining solutions, the proposed scheme effectively allocates the CWBAN resources in a fair-efficient manner. With the self-adaptability and real-time effectiveness, a well-balanced solution can be obtained while leveraging the full synergy of two bargaining solutions. In summary, the contributions of this paper are as follows:

(i) Bandwidth allocation algorithm based on the GGBS: motivated by the GGBS, the wireless bandwidth is allocated among WBANs to connect the cloud server. Depending on the current situation, bargaining parameters are dynamically decided in an online manner.

(ii) Computation capacity distribution algorithm based on the CBS: motivated by the CBS, the computation power of cloud server is distributed to each WBAN. 
This approach can investigate the potential benefit gained from practical cooperative bargaining method.

(iii) The synergy of two combined bargaining solutions: we explore the interaction of GGBS and CBS methods and jointly design an integrated dualphase cooperative game model to leverage the synergistic and complementary features of CWBAN services. The main idea of our dual-phase approach lies in its responsiveness to the reciprocal combination of different bargaining solutions.

(iv) Implementation practicality: under dynamic CWBAN environments, traditional cooperative bargaining solutions suffer from the inevitable burden of uncertainty. From the viewpoint of practical operations, we attempt to approximate the finest solution based on the step-by-step interactive fashion. This approach is generic and applicable for real-world CWBAN operations.

(v) Performance analysis: the major challenge of our proposed scheme is to try to strike the appropriate performance fairly and efficiently. Numerical simulation study shows that a timely effective solution is dynamically obtained based on the joint bargaining solutions.

1.2. Organization. The rest of this paper is organized as follows. In Section 2, we briefly discuss the existing protocols on cloud-assisted WBANs. Section 3 describes some preliminaries about the GGBS and the CBS and the CWBAN system infrastructure. Then, we formulate mathematical models to develop our proposed resource allocation algorithms. For readers' convenience, we also provide the primary steps of the proposed scheme. Experimental evaluation results are presented in Section 4. In this section, we verify the effectiveness of the proposed CWBAN control scheme while providing comparison with existing state-of-the-art protocols. Finally, in Section 5, we make some concluding remarks along with open issues and future work.

\section{Related Work}

Over the years, a lot of state-of-the-art research works on the CWBAN system have been conducted. Quwaider and Jararweh propose a new cloud-supported model to process large-scale WBAN data in order to detect the abnormal data [13]. Their main contributions are (i) introducing a scalable and cost-effective computing model, (ii) providing a scalable storage with a MapReduce based processing infrastructure, (iii) supporting mobile based WBANs system using cost-effective communication technologies, and (iv) introducing a high-speed computing infrastructure using the state-of-the-art MapReduce computing model. Finally, performance results show that applying MapReduce infrastructure in lower tire can significantly decrease the overall processing delay [13]. They also present another novel cloudlet-based system in WBANs [14]. The goal in paper [14] is to have a large scale of monitored data of WBANs to be available at the end user or to the service provider in reliable manner. This approach is fully supporting for WBANs system mobility using cost-effective communication technologies of Wi-Fi and cellular networks which are supported by WBANs and virtualized cloudlet systems. It is shown that the average power consumption and delay of the collected data are tremendously decreased by increasing the number of virtual machines and virtualized cloudlets [14].

The Multistage Bargaining based CWBAN (MBCWBAN) scheme is developed to provide the bargaining based resource allocation and price agreement in an environment of CWBAN system [15]. The MB-CWBAN scheme attempts to use the concept of bargaining and introduces a multistage Nash bargaining solution to reach a price agreement between the cloud server and the WBAN. By considering the ranking of the cloud servers and the physiological severity of each WBAN, this multistage bargaining method is an innovative extension of the general Nash bargaining solution. Therefore, the bargaining based negotiation process repetitively updates the minimum and maximum price bids of the WBANs and the cloud servers, respectively, after each stage, until they converge to a price agreement. Depending on the cost-effectiveness of the mapping from the perspective of a WBAN, the resource allocation process among the WBANs is developed according to their utility functions [15].

In [3], Misra and Samanta propose the Traffic Awareness based CWBAN (TA-CWBAN) scheme to identify the problem of traffic load minimization and selection of an optimal pricing policy for heterogeneous WBANs in a cloud-enabled platform. A cloud infrastructure provides its resources on requirements of WBANs in a ubiquitous manner, and each WBAN can access the cloud resource. This scheme leads to an overall resource optimization due to sharing of resources and reduction in the overall cost of usage. Based on the WBAN grouping, the TA-CWBAN attempts to minimize the computational complexity and traffic load in an emergency situation. According to the capacity, robustness, and capacity in terms of available resources, service delay, and pricing policy, the TA-CWBAN scheme focuses on the optimal mapping of WBANs to cloud servers. Simulation results exhibit a significant performance improvement in the CWBAN system [3].

The Resource Provisioning for CWBAN (RP-CWBAN) scheme presents a fast and robust cloud resource allocation model for WBANs to ensure the Quality of Service (QoS) [7]. Using the agent-based modeling and ontology, a novel resource allocation algorithm is developed for the CWBAN system. To address the problem of cloud resource allocation, this approach attempts to optimize the resource allocation model using mixed-integer linear programming method. The main contribution of the RP-CWBAN scheme is to maintain the QoS requirements of applications while processing and analyzing the huge data in real time. QoS requirements include low latency and network resource consumption and optimal utilization of computational recourses. According to a dynamic and fast resource scheduling technique for heterogeneous workloads in the 
CWBAN system, the advantages of RP-CWBAN scheme can be demonstrated in the simulation analysis [7].

The MB-CWBAN, TA-CWBAN, and RP-CWBAN schemes have introduced unique challenges to efficiently solve the CWBAN's control problem and have attracted a lot of attentions recently. Compared to these existing schemes in $[3,7,15]$, we demonstrate that the proposed scheme attains a better performance during the CWBAN system operations.

\section{The Proposed CWBAN Resource Allocation Scheme}

In this section, we explain preliminarily the basic ideas of GGBS and CBS and the infrastructure of CWBAN. Then, we apply these bargaining solutions to design a new dual-phase cooperative game model and develop a new control scheme, which can strike an appropriate resource allocation in the CWBAN system.

3.1. Multiutilitarian Bargaining Solutions. A cooperative bargaining problem consists of a set of game players and a feasible set of payoffs. Based on a common principle of rationality, most solutions of bargaining problems are consistent with the maximization of some ordering of the utility space. Until now, various solutions have been proposed based on slightly different assumptions about what properties are desired for the final agreement point. As a class of bargaining solutions, multiutilitarian bargaining solutions have been developed. They are rationalized by piecewise linear increasing social welfare functions defined on the players' utility gains. They depend on a set of weighted utilitarian criteria and on the decision rule used to combine them. Each multiutilitarian bargaining solution is associated with a probability measure over the set of weighted utilitarian rules and is derived as the expectation of this probability. These rules are characterized by the axioms, which are related to the issues of efficiency and fairness $[12,16]$. In this study, we introduce and characterize two bargaining solutions, which are the Generalized Gini Bargaining Solution (GGBS) and the Choquet Bargaining Solution (CBS), in the class of multiutilitarian bargaining solutions.

In 1994, Blackorby et al. provided the concept of GGBS that can be rationalized by the generalized Gini social welfare functions. The GGBS can be considered as a compromise between the two polar bargaining solutions, namely, the utilitarian and the egalitarian solutions. In general, the GGBS can be regarded as compelling social choice rules [17]. In 2000, Ok and Zhou proposed the concept of CBS. The CBS corresponds to orderings that can be represented by social welfare functions derived from Choquet integrals with respect to a monotonic capacity. The CBS contains the GGBS as special cases. More precisely, the CBS can be rationalized by social welfare functions, which are strictly increasing and are linear on every rank-ordered subset. The CBS differs from the GGBS in that it does not need to be anonymous and its representing social welfare function is not necessarily quasi-concave. All weighted utilitarian bargaining solutions are the CBS that can be rationalized by a linear combination of the utilitarian and the max-max social welfare functions [18].

To characterize the basic concepts of GGBS and CBS, we preliminarily define some mathematical expressions. Let $\mathbb{R}\left(\mathbb{R}_{+}, \mathbb{R}_{++}\right)$denote the set of all (nonnegative, positive) real numbers and let $\mathbb{R}^{\mathrm{n}}\left(\mathbb{R}_{+}^{n}, \mathbb{R}_{++}^{n}\right)$ be the $n$-fold Cartesian product of $\mathbb{R}\left(\mathbb{R}_{+}, \mathbb{R}_{++}\right)$. We use conventional notation for comparison of vectors: $\mathbf{x} \geq \mathbf{y}$ means that $x_{i} \geq y_{i}$ for all $i=1,2$, $\ldots, n, \mathbf{x}>\mathbf{y}$ indicates that $\mathbf{x} \geq \mathbf{y}$ and $\mathbf{x} \neq \mathbf{y}$, and $\mathbf{x} \gg \mathbf{y}$ means $x_{i}>\mathrm{y}_{\mathrm{i}}$ for all $i=1,2, \ldots, n$. We denote the scalar product of the vectors $\mathbf{x}, \mathbf{y} \in \mathbb{R}^{\mathrm{n}}$; that is, $\mathbf{x} \cdot y=\sum_{i=1}^{n}\left(x_{i} \times y_{i}\right)[12,16]$. $N=\{1,2, \ldots, n\}$ is a set of $n$ game players and $2^{N}$ is the set of all possible subsets of $N$. An $n$-player bargaining problem can be described by a set of feasible utility vectors, $S \subseteq \mathbb{R}_{+}^{n}$, where we assume that $S$ is convex, compact, and comprehensive and that there exists $\mathbf{x} \in S \cap \mathbb{R}_{++}^{n}$. The set of $n$-player bargaining problems is denoted by $\sum$. A bargaining solution is a correspondence $F: \sum \longrightarrow \mathbb{R}_{+}^{n}$ such that $\varnothing \neq F(S) \subseteq S$ for all $S \in \sum$. Notice that $F$ is allowed to yield multiple outcomes to a problem. Some simple bargaining solutions are given as follows [12].

(i) The egalitarian bargaining solution $\mathbf{E}(\mathbf{S})$ : for each $S \in \sum, E(S)=\left\{\mathbf{x} \in S \mid x_{i}=x_{j}\right.$ or all $i, j=1,2, \ldots, n$, and there is no $\mathbf{y} \in S$ such that $\mathbf{y} \gg \mathbf{x}\}$.

(ii) The utilitarian bargaining solution $\mathbf{U}(\mathbf{S})$ : for each $S \in \sum, U(S)=\left\{\mathbf{x} \in S \mid \sum_{i=1}^{n} x_{i} \geq \sum_{i=1}^{n} y_{i}\right.$ for all $\left.\mathbf{y} \in S\right\}$ with $\in \mathbb{R}_{++}^{n}$.

(iii) The multiutilitarian bargaining solution $\mathbf{M}(\mathbf{S})$ : for each $S \in \sum, \quad M(S)=\left\{\mathbf{x} \in S \mid \max U^{l}(\mathbf{x})\right.$ where $\left.U^{l}(\mathbf{x})=\psi^{l} \cdot \mathbf{x}\right\}$ with $\psi^{l} \in \mathbb{R}_{++}^{\mathbf{n}}$ for $l \in\{1,2, \ldots, k\}$.

(iv) The weighted utilitarian ( $\boldsymbol{\lambda}$-utilitarian) bargaining solution $\mathbf{U}_{\lambda}$ : for each $S \in \sum, U_{\lambda}(S)=\{\mathbf{x} \in S$ $\mid \sum_{i=1}^{n}\left(\lambda_{i} \times x_{i}\right) \geq \sum_{i=1}^{n}\left(\lambda_{i} \times y_{i}\right)$ for all $\left.\mathbf{y} \in S\right\}$ with $\lambda \in$ $\mathbb{R}_{++}^{n}$.

$\mathbf{U}^{\mathbf{l}}(\mathbf{x})$ is a social welfare function that is increasing and linear in every rank-ordered subset of the set of $n$-dimensional vectors with nonnegative components. Note that the weighted utilitarian solution is a particular case of multiutilitarian solution in $\mathbb{R}_{+}^{\mathbf{n}}[12]$.

Now, we explain the idea of GGBS. Let $\pi: \mathbf{N} \longrightarrow \mathbf{N}$ denote a permutation function, in which $\pi(\mathbf{i})=\mathbf{j}$ means that the $\mathbf{i}^{\text {th }}$ component of the $\mathbf{n}$-dimensional permuted vector is $\mathbf{j}$. Let $\mathbf{x}_{\boldsymbol{\pi}}$ be the $\boldsymbol{\pi}$-permutation of vector $\mathbf{x} \in \mathbb{R}^{\mathbf{n}}$; $\mathbf{x}_{\boldsymbol{\pi}}=\left(\mathbf{x}_{\boldsymbol{\pi}(1)}, \mathbf{x}_{\boldsymbol{\pi}(2)}, \ldots, \mathbf{x}_{\boldsymbol{\pi}(\mathbf{n})}\right)$. The set of all possible permutation functions is denoted by $\prod$. For $\Upsilon=\left\{\Upsilon_{1}, \Upsilon_{2}, \ldots, \Upsilon_{\mathbf{n}}\right\} \in \mathbb{R}_{+}^{\mathbf{n}}$, such that $\Upsilon_{1} \geq \Upsilon_{2} \geq$ $\ldots \geq \Upsilon_{\mathbf{n}}>0, \mathbf{G}_{Y}: \mathbb{R}^{\mathbf{n}} \longrightarrow \mathbb{R}$ represents the generalized Gini ordering $[12,16,17]$ :

$$
G_{Y}(\mathbf{x})=\sum_{i=1}^{n}\left(\Upsilon_{i} \times x_{(i)}\right),
$$

where $\left(x_{(1)}, x_{(2)}, \ldots, x_{(n)}\right)$ denotes a rank-ordered permutation vector of $\mathbf{x} \in \mathbb{R}_{+}^{n}$; that is, $x_{(1)} \leq x_{(2)} \leq, \ldots, \leq x_{(n)}$. Let $\Delta^{n}$ be the simplex in $\mathbb{R}_{+}^{\mathrm{n}}$ and $\Delta^{n}=\left\{\Upsilon \in \mathbb{R}_{+}^{\mathrm{n}} \mid \Upsilon \cdot 1^{n}=1\right\}$. For 
each $S \in \sum$, the GGBS with $G_{Y}(\mathbf{x})$ is defined as follows $[12,16,17]$ :

$$
\begin{aligned}
\mathrm{GGBS}_{\Upsilon}(S) & =\arg \max _{x \in S} G_{\Upsilon}(x) \\
& =\arg \max _{x \in S} \sum_{i=1}^{n}\left(\Upsilon_{i} \times x_{(i)}\right), \quad \text { s.t., } \Upsilon \in \Delta^{n} .
\end{aligned}
$$

Now, we explain the idea of CBS. Let $\mathscr{V}$ be a monotonic real-valued set function on $2^{N}$ with $\mathscr{V}(\varnothing)=0$. We suppose that $\mathscr{V}$ is 1 -normalized; that is, $\mathscr{V}(N)=1$, and it is monotonic if $\mathscr{V}(K)<\mathscr{V}(L)$ for all $K \subset L$. Associated with the monotonic feature, the Choquet social welfare function is defined as $[12,16,18]$

$$
\begin{aligned}
\mathscr{W}_{\mathscr{V}}(\mathbf{x})= & \sum_{i=1}^{n}([\mathscr{V}(\{(i),(i+1), \ldots,(n)\}) \\
& \left.-\mathscr{V}(\{(i+1), \ldots,(n)\})] \times x_{(i)}\right),
\end{aligned}
$$

where $\left(x_{(1)}, x_{(2)}, \ldots, x_{(n)}\right)$ denotes a rank-ordered permutation of $\mathbf{x} \in \mathbb{R}_{+}^{\mathbf{n}}$; that is, $x_{(1)} \leq x_{(2)} \leq, \ldots, \leq x_{(n)}$. For each $S \in \sum$, the CBS, $\mathscr{C}_{\mathscr{V}}(S)$, is defined as follows $[12,16,18]$ :

$$
\mathscr{C}_{\mathscr{V}}(S)=\arg \max _{\mathbf{x} \in S} \mathscr{W}_{\mathscr{V}}(\mathbf{x})
$$

To examine the axiomatic basis of the GGBS and CBS, we denote by $\mathrm{PO}(\mathrm{S})$ the set of all strongly Pareto-optimal points of $S$; $\mathrm{PO}(\mathrm{S})=\{x \in S \mid$ there exists no $y \in S, y>x\}$ for each $S \in \Sigma$. Let co $(A)$ denote the convex hull of the set $A$ in $\mathbb{R}^{\mathrm{n}}$, co $(A)=\left\{\mathbf{z} \in \mathbb{R}^{n} \mid \mathbf{z}=\alpha \cdot \mathbf{x}+(1-\alpha) \cdot \mathbf{y}, \mathbf{x}, \mathbf{y} \in A, \alpha \in[0,1]\right\}$, let $\operatorname{cch}(A)$ denote the convex and comprehensive hull of $A$, $\operatorname{cch}(A)=\left\{\mathbf{y} \in \mathbb{R}^{n} \mid \mathbf{y} \leq \mathbf{z}, \mathbf{z} \in \operatorname{co}(A)\right\}$, and let int $(A)$ denote the relative interior of $A[12,16]$. The axioms involved in the characterization of GGBS and CBS are stated below.

Strong Pareto-optimality (SPO): for all $S \in \Sigma, F(S) \subseteq$ PO (S).

Arrow's choice axiom (ACA): for all $S, T \in \Sigma$, if $T \subseteq S$ and $F(S) \cap T \neq \varnothing$, then $F(T)=F(S) \cap T$.

Continuity (CON): for all $S \in \Sigma$ and $x \in S$, if there exists a sequence $S_{m}$ such that (i) $\left|F\left(S_{m}\right)\right|=1$ for all $m$, (ii) $S_{m} \longrightarrow S$ (in Hausdorff topology), and (iii) $F\left(S_{m}\right) \longrightarrow\{x\}$, then $\mathbf{x} \in F(S)$.

Coregional invariance axiom (Co-INV): there exists a class of equivalence, $\Omega$, such that, for all $S \in \Sigma$, $\mathbf{x} \in F(S)$, and $\mathbf{y} \in \mathbb{R}^{n}$, with $\operatorname{cch}(S+\mathbf{y}) \in \Sigma$, if $\mathrm{PO}(S)$ and $\mathrm{PO}(S+\mathbf{y})$ are $\Omega$-coregional, then $\mathbf{x}+\mathbf{y} \in F(c c h(S+\mathbf{y}))$. Compromisability (COM): for all $S \in \Sigma,|F(S)| \neq 2$.

Anonymity axiom (A): for all $S \in \Sigma, \mathbf{x} \in F(S)$, and $x_{\pi} \in F\left(\left\{y_{\pi} \mid \mathbf{y} \in S\right\}\right)$ for any $\pi \in \Pi$.

The SPO axiom states that the solution outcome should exhaust all gains from cooperation. The ACA axiom requires that choices be independent of contraction of the choice set. The CBS satisfies the axioms of SPO, ACA, CON, Co-INV, and COM. The A axiom specially characterizes the GGBS. Therefore, the GGBS satisfies the axioms of SPO, ACA, CON, Co-INV, COM, and A $[12,16]$.
3.2. Dual-Phase Bargaining Model for the CWBAN System. In case of modern ubiquitous healthcare environments, a new architectural element of cloud, called cloudlet, is introduced to extend the traditional cloud computing infrastructure. A cloudlet is a mobility-enhanced small-scale cloud server that is located at the edge of the Internet. The cloudlet can provide powerful computing resources to WBANs with lower latency. In this study, our CWBAN system is designed based on the cloudlet. To contact the cloudlet through wireless communications, each individual WBAN has a single entity called Central Processing Coordinator (CPC). In our CWBAN system platform, a set of WBANs are present in an area, which are covered by the cloudlet $\mathscr{C}$. The $\mathrm{CPC}$ of each WBAN asks $\mathscr{C}$ for both communication and computation resources.

Our dual-phase bargaining game model consists of two resource allocation algorithms. In the first phase, we assume that individual CPCs are game players, and they attempt to maximize their payoffs through the communication resource allocation process. In the second phase, we mainly focus on addressing the computation resource allocation problem among offloaded computation tasks. To share optimally the limited resources of CWBAN system, the communication and computation resource allocation algorithms are designed based on the ideas of GGBS and CBS. Under dynamically changing CWBAN system environments, it is suitable for the ultimate practical WBAN system operations. To obtain a fair-efficient solution, the allocation decision process is formulated as a cooperative bargaining game $\mathbb{G}=\left\{\mathbb{T}=\left\{\Gamma_{1}, \ldots, \Gamma_{n}\right\}, \mathbb{P}_{\Gamma}=\left\{\mathscr{P}_{1}^{\Gamma}, \mathscr{P}_{2}^{\Gamma}, \ldots, \mathscr{P}_{m}^{\Gamma}\right\}\right.$, $\left.\left\{U_{\Gamma}, \mathscr{U}_{\mathscr{V}}\right\}, \mathbb{A}_{\Gamma}=\left\{\ldots \mathscr{A}_{k}^{\Gamma} \ldots\right\},\left\{\mathscr{C}, \mathscr{P}_{\mathscr{C}}, \mathfrak{S}_{\mathscr{C}}\right\}, \mathbb{S}=\{\mathbf{x}, \mathbf{y}\}\right\}:$

(i) $\mathbb{T}$ is the set of CPCs, where $\Gamma_{1 \leq i \leq n}$ is the CPC of $i^{\text {th }}$ WBAN.

(ii) $\mathbb{P}_{\Gamma}$ is the set of $\Gamma$ 's possible price policies for CWBAN services, where $\mathscr{P}_{1 \leq j \leq m}^{\Gamma}$ represents the $j^{\text {th }}$ price policy of $\Gamma$ to get the communication and computation resources.

(iii) $U_{\Gamma}$ is the $\Gamma$ 's utility function, and $\mathscr{U}_{\mathscr{V}}$ is the social welfare function. Each function maps the player's satisfaction to a real number, which represents the resulting payoff in the game $\mathbb{G}$.

(iv) $\mathbb{A}_{\Gamma}$ is the set of $\Gamma$ 's application tasks, which are generated from the $\Gamma$ 's WBAN and ask for offloading services.

(v) The cloudlet $\mathscr{C}$ has its communication resource, that is, $\mathscr{P}_{\mathscr{C}}$, and computation capacity, that is, $\mathfrak{E}_{\mathscr{C}}$, to provide offload services.

(vi) Bargaining solution set $\mathbb{S}$ consists of two $n$-dimensional vectors $\mathbf{x}$ and $\mathbf{y}$, which correspond to the communication and computation resource allocations for each $\Gamma$.

At the first phase, $\mathscr{P}_{\mathscr{C}}$ of CWBAN system is adaptively allocated to each CPC according to the idea of GGBS. To quantify the communication service satisfaction, the payoff of player $\Gamma_{i}$ with $\mathbb{P}_{\Gamma_{i}}$ for $\mathbb{A}_{\Gamma_{i}} \cdot\left(U_{\Gamma_{i}}\left(\mathbb{P}_{\Gamma_{i}}, \mathbb{A}_{\Gamma_{i}}\right)\right)$ can be derived as follows: 


$$
\begin{aligned}
& U_{\Gamma_{i}}\left(\mathbb{P}_{\Gamma_{i}}, \mathbb{A}_{\Gamma_{i}}\right)=x_{\Gamma_{i}} \times\left(\Gamma-\frac{1}{\exp \left(\mu \times x_{\Gamma_{i}} / \mathscr{M}\left(\mathbb{A}_{\Gamma_{i}}\right)\right)}\right)^{X}, \\
& \text { s.t., }\left\{\begin{array}{l}
\mathscr{M}\left(\mathbb{A}_{\Gamma_{i}}\right)=\sum_{\mathscr{A}_{k}^{\Gamma_{i}} \in \mathbb{A}_{\Gamma_{i}}} \mathscr{R}_{\mathscr{P}_{\mathscr{G}}}\left(\mathscr{A}_{k}^{\Gamma_{i}}\right), \\
\mathscr{X}=\beta+\log \left(1+\left(\frac{\sum_{\mathscr{A}_{k}^{\Gamma_{i}} \in \mathbb{A}_{\Gamma_{i}}} \mathcal{S}_{\mathbb{P}_{\Gamma_{i}}}\left(\mathscr{A}_{k}^{\Gamma_{i}}\right)}{\left|\mathbb{A}_{\Gamma_{i}}\right|}\right)\right),
\end{array}\right.
\end{aligned}
$$

where $\boldsymbol{\mu}$ and $\boldsymbol{\beta}$ are the control parameters for $\mathbf{U}_{\Gamma_{\mathrm{i}}}\left(\mathbb{P}_{\Gamma_{\mathrm{i}}}, \mathbb{A}_{\Gamma_{\mathrm{i}}}\right)$ and $\mathbf{x}_{\Gamma_{\mathrm{i}}}$ is the total amount of allocated communication resource for $\Gamma_{\mathrm{i}}$. $\mathscr{R}_{\mathscr{B}_{\mathscr{C}}}\left(\mathscr{A}_{k}^{\Gamma_{i}}\right)$ is the requested amount of communication resource for $\mathscr{A}_{k}^{\Gamma_{i}}$, and $\mathcal{S}_{\mathbb{P}_{\Gamma_{i}}}\left(\mathscr{A}_{k}^{\Gamma_{i}}\right)$ is the price policy of $\mathscr{A}_{k}^{\Gamma_{i}}$. $\left|\mathbb{A}_{\Gamma_{\mathrm{i}}}\right|$ is the cardinality of the set $\mathbb{A}_{\Gamma_{\mathrm{i}}}$.
Each CPC periodically reports its $\mathscr{M}\left(\mathbb{A}_{\Gamma}\right)$ and $\mathscr{X}$ information to the cloudlet server $\mathscr{C}$. At this time, the communication resource, that is, wireless bandwidth, should be dynamically allocated to the corresponding CPCs. In this study, we adopt the method of GGBS to solve the bandwidth allocation problem in the CWBAN system. To obtain the GGBS, we should define the permutation vector $\mathbf{x}_{\boldsymbol{\pi}}$ and the simplex $\mathscr{A}$ in $\mathbb{R}_{+}^{\mathbf{n}}$. In the proposed scheme, the order of $\mathbf{x}_{(\mathbf{i})}$ in $\mathbf{x}_{\boldsymbol{\pi}}$ and the order of $\mathscr{A}_{\mathbf{i}}$ in $\mathscr{A}$ are decided based on each $\Gamma$ 's quantitative concern and qualitative attention. The rationale behind this decision mechanism is a mix of the egalitarian and utilitarian principles. Therefore, the combination of $\mathbf{x}_{\boldsymbol{\pi}}$ and $\mathscr{A}$ is inequality averse but includes weighted utilitarian criteria to achieve compromise bargaining solution. Based on the amount of requested communication resource and the price policy, the relative importance of $\Gamma_{\mathbf{i}} \cdot\left(\mathrm{I}_{\Gamma_{\mathrm{i}}}\right)$ can be defined as follows:

$$
I_{\Gamma_{i}}=\log \left(1+\frac{m_{\Gamma_{i}}}{\mathrm{M}_{\Gamma}}\right), \quad \text { s.t., }\left\{\begin{array}{l}
m_{\Gamma_{i}}=\sum_{\mathscr{A}_{k}^{\Gamma_{i}} \in \mathbb{A}_{\Gamma_{i}}}\left(\mathcal{S}_{\mathbb{P}_{\Gamma_{i}}}\left(\mathscr{A}_{k}^{\Gamma_{i}}\right) \times \mathscr{R}_{\mathscr{P}_{\mathscr{G}}}\left(\mathscr{A}_{k}^{\Gamma_{i}}\right)\right), \\
\mathrm{M}_{\Gamma}=\max _{\Gamma_{i} \in \mathbb{T}} m_{\Gamma_{i}} .
\end{array}\right.
$$
follows:

According to (2), we can get the simplex vector $\mathbf{V}_{\Gamma}$ as

$$
\mathbf{V}_{\Gamma}=\left(v_{\Gamma_{1}}, v_{\Gamma_{2}}, \ldots, v_{\Gamma_{n}}\right), \quad \text { s.t., } v_{\Gamma_{i}}=\frac{I_{\Gamma_{i}}}{\sum_{\Gamma_{l} \in \mathbb{T}} I_{\Gamma_{l}}} .
$$

Using the vector $\mathbf{V}_{\Gamma}$, we define the vector $\Upsilon_{\Gamma}=\left\{\Upsilon_{1}, \Upsilon_{2}, \ldots, \Upsilon_{n}\right\}$ based on the one-to-one mapping with $\mathbf{V}_{\Gamma}$ while satisfying the order $\Upsilon_{1} \geq \Upsilon_{2} \geq \cdots \geq \Upsilon_{n}>0$. The $\pi$-permutation of vector $\mathbf{x}_{\pi(\Gamma)}$ is obtained based on the $m_{\Gamma_{i}}$ value of each $\Gamma$. Therefore, $\mathbf{x}_{\pi(\Gamma)}=\left(x_{\left(\Gamma_{1}\right)}, x_{\left(\Gamma_{2}\right)}, \ldots, x_{\left(\Gamma_{n}\right)}\right)$ denotes a rank-ordered permutation vector of $\mathbf{x}_{\pi(\Gamma)} \in \mathbb{R}_{+}^{n}$, where $x_{\left(\Gamma_{1}\right)} \leq x_{\left(\Gamma_{2}\right)} \leq, \ldots, \leq x_{\left(\Gamma_{n}\right)}$. Based on $\mathbf{I}_{\Gamma}$ and $\mathbf{x}_{\pi(\Gamma)}$, the GGBS for each $S \in \sum$ is finally given by

$$
\operatorname{GGBS}_{\mathbf{I}_{\Gamma}}(S)=\arg \underset{\mathbf{x} \in S}{\max } \sum_{i=1}^{n}\left(\Upsilon_{i} \times x_{\left(\Gamma_{i}\right)}\right) \text {, s.t., } \mathbf{I}_{\Gamma} \in \Delta^{n} .
$$

Like the communication resource, the limited computation resources of $\mathscr{C}$ can negatively impact the CWBAN performance. Hence, $\mathscr{C}$ needs a viable solution to effectively distribute the cloud computing power to the offloaded tasks. In this study, we adopt the method of CBS to solve this computation resource allocation problem in the CWBAN system. In the proposed scheme, we assume that each $\Gamma$ 's computation task can be offloaded to $\mathscr{C}$. At this time, $\mathscr{C}$ should distribute its computation power $\mathfrak{C}_{\mathscr{C}}$ to each $\Gamma$ while considering the system efficiency and fairness. Based on the amount of requested computation resource and the price policy, we define the 1-normalized monotonic set function $\mathscr{V}$ as follows:

$$
\begin{aligned}
& \mathscr{V}\left(\left\{\Gamma_{i}, \Gamma_{i+1}, \ldots, \Gamma_{n}\right\}\right)=\ln \left(\eta+\left(\frac{\mathscr{X}\left(\Gamma_{i}\right)}{\mathcal{Z}(\mathbb{T})}\right)\right)^{\psi}, \\
& \text { s.t., }\left\{\begin{array}{l}
\mathscr{X}\left(\Gamma_{i}\right)=\sum_{j=i}^{n} \sum_{\mathscr{A}_{k}^{\Gamma_{j}} \in \mathbb{A}_{\Gamma_{j}}} r_{\Gamma_{j}}\left(\mathbb{A}_{\Gamma_{j}}\right), \\
\mathscr{Z}(\mathbb{T})=\sum_{j=1}^{n} \sum_{\mathscr{A}_{k}^{\Gamma_{j}} \in \mathbb{A}_{\Gamma_{j}}} r_{\Gamma_{j}}\left(\mathbb{A}_{\Gamma_{j}}\right), \\
\mathscr{r}_{\Gamma_{j}}\left(\mathbb{A}_{\Gamma_{j}}\right)=\mathcal{S}_{\mathbb{P}_{\Gamma_{j}}}\left(\mathscr{A}_{k}^{\Gamma_{j}}\right) \times \mathscr{R}_{\mathbb{C}_{\mathscr{G}}}\left(\mathscr{A}_{k}^{j}\right),
\end{array}\right.
\end{aligned}
$$

where $\eta$ and $\psi$ are the control parameters for $\mathscr{V}\left(\left\{\Gamma_{i}, \Gamma_{i+1}, \ldots, \Gamma_{n}\right\}\right)$ and $\mathscr{R}_{\mathfrak{C}_{\mathscr{E}}}\left(\mathscr{A}_{k}^{j}\right)$ is the requested amount of computing power for $\mathscr{A}_{k}^{\Gamma_{i}}$. It is assumed that $\mathscr{V}(\varnothing)=0$ and $\mathscr{V}(N)=1$. Based on the set function $\mathscr{V}$, the Choquet social welfare function $\mathscr{U}_{\mathscr{V}}(\mathbf{y})$ can be defined as follows:

$$
\begin{aligned}
\mathscr{U}_{\mathscr{V}}(\mathbf{y})= & \sum_{i=1}^{n}\left(\left[\mathscr{V}\left(\left\{\left(\Gamma_{i}\right),\left(\Gamma_{i+1}\right), \ldots, \Gamma_{n}\right\}\right)-\left(\left\{\left(\Gamma_{i+1}\right), \ldots, \Gamma_{n}\right\}\right)\right]\right. \\
& \left.\times y_{(i)}\right) .
\end{aligned}
$$

The $\pi$-permutation of vector $\mathbf{y}_{\pi(\Gamma)}$ is obtained based on the $\mu_{\Gamma_{j}}\left(\mathbb{A}_{\Gamma_{j}}\right)$ value of each $\Gamma$. Therefore, the $\pi$-permutation of vector $\mathbf{y}_{\boldsymbol{\pi}(\Gamma)}=\left(y_{\left(\Gamma_{1}\right)}, y_{\left(\Gamma_{2}\right)}, \ldots, y_{\left(\Gamma_{n}\right)}\right)$ also denotes the 
rank-ordered permutation vector of $\mathbf{y}_{\pi(\Gamma)} \in \mathbb{R}_{+}^{n}$, where $y_{\left(\Gamma_{1}\right)} \leq y_{\left(\Gamma_{2}\right)} \leq, \ldots, \leq y_{\left(\Gamma_{n}\right)}$. Finally, the CBS, $\mathscr{C}_{\mathscr{V}}(S)$, is given by

$$
\mathscr{C}_{\mathscr{V}}(S)=\arg \max _{\mathbf{y} \in S} \mathscr{U}_{\mathscr{V}}(\mathbf{x}) \mathscr{R}
$$

3.3. The Main Steps of Proposed Resource Allocation Scheme. Our work attempts to identify the resource allocation problem for heterogeneous WBANs in a cloud-enabled platform. The cloudlet infrastructure can provide cloud resources to a WBAN in ubiquitous manner. Thus, it leads to an overall resource optimization problem for the sharing of limited resources. Commonly, cooperative game theory has been used a lot for the resource allocation problems. In this paper, our proposed scheme mainly tackles the research challenges on how to efficiently allocate the CWBAN system's communication and computation resources in accordance with two cooperative bargaining solutions: the GGBS and CBS. For the communications of WBANs, we emphasize the anonymity of each WBAN. Therefore, the method of GGBS is applied to design the communication resource allocation component. On the other hand, the idea of CBS is adopted to develop the computing resource allocation component by considering the tradeoff between egalitarian and utilitarian characteristics. These two components are executed based on the step-by-step interactive fashion and work together to leverage the synergistic and complementary features of CWBAN services. Therefore, the proposed dual-phase bargaining game process is sequentially repeated over the time period to approximate a fine solution through a step-by-step approach. The main steps of the proposed scheme can be described as follows:

Step 1: control parameters and system factors are given by the simulation scenario in Section 4 (refer to Table 1).

Step 2: as a game player, each individual CPC has its own price policy for each application task. At each time iteration, CPCs request the resources, that is, wireless bandwidth and computing power, based on their generated application tasks.

Step 3: the first phase game process is designed for the allocation problem of communication resource. In this game model, the CPCs' payoffs $\left(U_{\Gamma}\right)$ and the relative importance $\left(I_{\Gamma}\right)$ are calculated according to (5) and (6).

Step 4: using equation (6), we calculate the simplex vector $\mathbf{V}_{\Gamma}$. Then, the ordering vectors $\Upsilon_{\Gamma}$ and $\mathbf{x}_{\pi(\Gamma)}$ are obtained based on the $\mathbf{V}_{\Gamma}$ and $m_{\Gamma}$ values.

Step 5: using $\Upsilon_{\Gamma}$ and $\mathbf{x}_{\pi(\Gamma)}$, the GGBS is finally obtained according to (8).

Step 6: the second phase game process is designed for the allocation problem of computation resource. According to equation (9), the 1-normalized set function $\mathscr{V}$ is defined.

Step 7: based on the set function $\mathscr{V}$, the Choquet social welfare function $\mathscr{U}_{\mathscr{V}}(\mathbf{y})$ is obtained according to (10).
Step 8: based on $\mathscr{U}_{\mathscr{V}}(\mathbf{y})$, the CBS is finally obtained according to (11).

Step 9: constantly, the CWBAN systems are selfmonitoring the current system situation, and proceed to Step 2 for the next dual-phase game iteration. The proposed resource allocation scheme is repeatedly operated over time.

\section{Performance Evaluation}

In this section, we compare the performance of the proposed scheme with other existing protocols. As mentioned in Section 2, we select the MB-CWBAN scheme [15], the TACWBAN scheme [3], and the RP-CWBAN scheme [7]; these existing protocols are recently published novel schemes for the cloud-assisted WBAN system. Through the simulation analysis, we can confirm the performance superiority of our approach. The assumptions of our simulation environment are as follows:

(i) The simulated CWBAN system consists of one cloudlet and five WBANs, that is, $\mathbb{T}=\left\{\Gamma_{1}, \Gamma_{2}, \Gamma_{3}, \Gamma_{4}, \Gamma_{5}\right\}$.

(ii) The process for application task generations is Poisson with rate $\lambda$ (tasks/s), and the range of offered service load was varied from 0 to 3.0.

(iii) The number of price policies is 6 , and each policy, that is, $\mathscr{P}_{1 \leq l \leq 6}^{\Gamma} \in\{1,1.2,1.4,1.6,1.8,2\}$, represents a price per one bit for communication resource or one FLOPS for computation resource.

(iv) The price policy is decided based on the type of each application task.

(v) System performance measures obtained on the basis of 100 simulation runs are plotted as a function of the offered task load.

(vi) $\mathscr{P}_{\mathscr{C}}$ and $\mathfrak{夭}_{\mathscr{C}}$ are assumed as the cloudlet $\mathscr{C}$ 's communication capacity and computing powers. They are 10 Tbps and 10 TFLOPS, respectively.

(vii) Performance measures obtained are normalized WBAN payoff ratio, resource utilization, and fairness among WBANs.

(viii) The allocated communication and computation resources for each $\Gamma$ are distributed to individual application tasks based on the utilitarian bargaining solution.

(ix) For simplicity, we assume the absence of physical obstacles in the cloudlet-WBAN communications.

Figure 1 shows the performance comparison of each scheme in terms of normalized WBAN payoff ratio. In this work, the payoff ratio is measured as the utility measurement of communication services. Therefore, communication payoffs of WBANs are combined and normalized for fair comparisons. From the viewpoint of cooperative game theory, the payoff maximization is one of the most critical aspects. In the simulation results, it is observed that the performance of our proposed scheme is comparatively better 
TABLE 1: System parameters used in the simulation experiments.

\begin{tabular}{|c|c|c|c|}
\hline Parameter & Value & \multicolumn{2}{|c|}{ Description } \\
\hline$n$ & 5 & \multicolumn{2}{|c|}{ The number of wireless body area networks } \\
\hline$m$ & 6 & \multicolumn{2}{|c|}{ The number of possible price policies of each CPC } \\
\hline$\mu$ & 1 & \multicolumn{2}{|c|}{ The control parameter for the payoff of $\Gamma$} \\
\hline$\beta$ & 1 & \multicolumn{2}{|c|}{ The control parameter for the payoff of $\Gamma$} \\
\hline$\eta$ & 1 & \multicolumn{2}{|c|}{ The control parameters for the function $\mathscr{V}$} \\
\hline$\psi$ & 1.4 & \multicolumn{2}{|c|}{ The control parameters for the function $\mathscr{V}$} \\
\hline Application type & Price policy & Communication amount & Computation amount \\
\hline $\mathscr{A}_{k} \in \mathrm{I}$ & 1.0 & $50 \mathrm{Gbps}$ & 30 GFLOPS \\
\hline $\mathscr{A}_{k} \in \mathrm{II}$ & 1.2 & 40 Gbps & 40 GFLOPS \\
\hline $\mathscr{A}_{k} \in$ III & 1.4 & 30 Gbps & 90 GFLOPS \\
\hline $\mathscr{A}_{k} \in \mathrm{IV}$ & 1.6 & 25 Gbps & 45 GFLOPS \\
\hline $\mathscr{A}_{k} \in \mathrm{V}$ & 1.8 & 45 Gbps & 25 GFLOPS \\
\hline $\mathscr{A}_{k} \in \mathrm{VI}$ & 2.0 & 60 Gbps & 50 GFLOPS \\
\hline
\end{tabular}

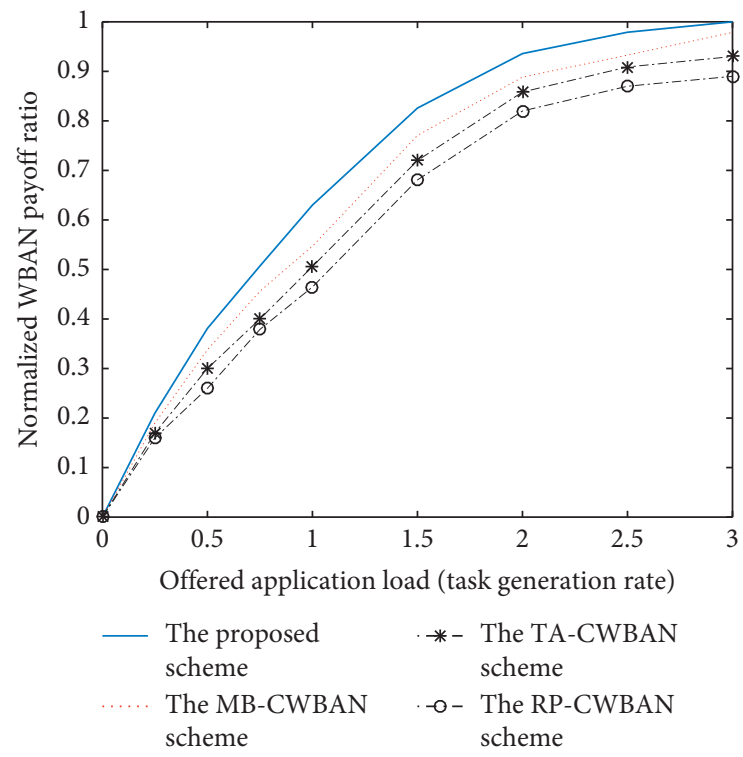

Figure 1: Normalized WBAN payoff ratio.

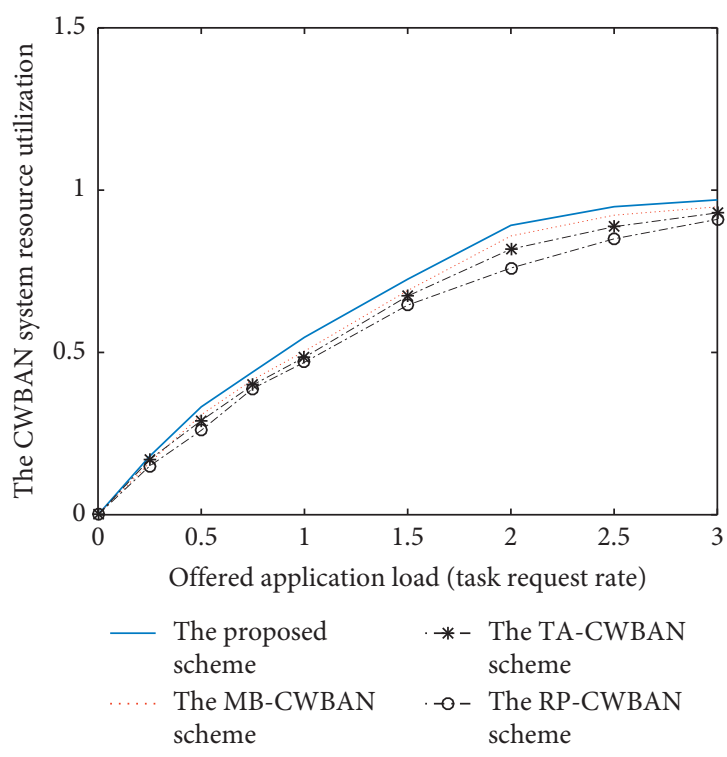

FIgURE 2: The CWBAN system resource utilization. than the existing protocols. This is due to our GGBS based approach, which can efficiently share the CWBAN communication resource; this approach effectively addresses the limited wireless bandwidth allocation problems among CPCs.

Figure 2 represents the resource utilization of each CWBAN control scheme. In this work, the resource utilization is measured to combine the usage rates of communication and computation resources and normalized them for fair comparisons. The curves shown in Figure 2 are very similar to the performance trend in Figure 1. In general, the higher payoff can be obtained based on the efficient resource usability. For low application load rates, the resource utilization is virtually the same as for the four schemes. However, as the application load rate increases, the resource utilization of our proposed scheme is better than the other schemes. According to the step-by-step sequential resource allocation mechanism, the GGBS and the CBS adaptively handle the CWBAN resource allocation problem based on the current system environment and make decisions on how to efficiently use the limited resources. Therefore, our proposed scheme can maintain a better resource utilization under various application load intensities.

Figure 3 depicts the fairness of CWBAN System. Fairness is a prominent and highly desirable property for the operation of multiple WBANs. To characterize the fairness notion, we follow Jain's fairness index [19], which has been frequently used to measure the fairness in the network resource allocation. In this work, we combine Jain's fairness indexes of communication and computation resource allocations and normalize them for fair comparisons. The main novelty of GGBS and the CBS is to distribute the resource in a fair-efficient manner. Therefore, in our proposed scheme, the actual outcome is fairly dealt out among multiple WBANs. As shown in Figure 3, the profit-sharing fairness in our approach is distinctly better than the existing schemes in $[3,7,15]$, which are designed as lopsided and one-way methods and do not effectively consider the fairness issue. 


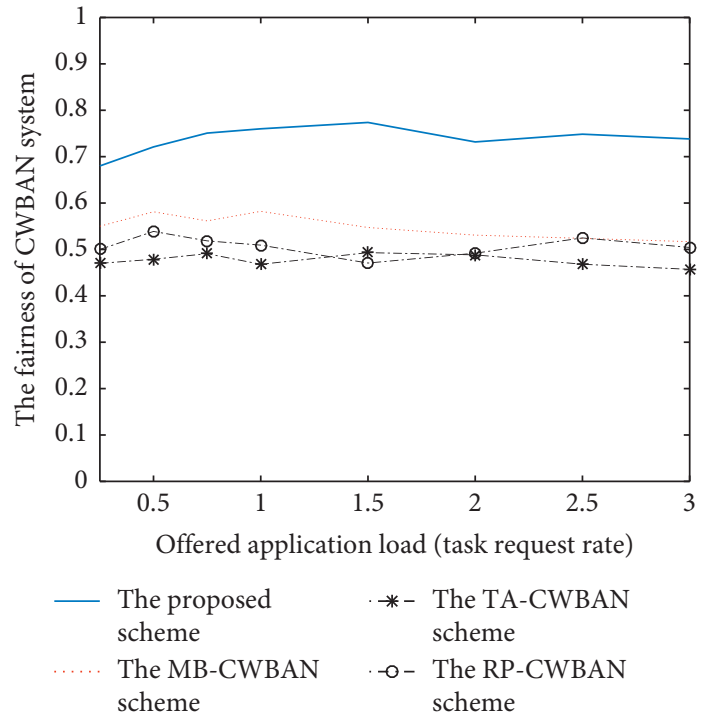

Figure 3: The fairness of CWBAN System.

The simulation results shown in Figures 1-3 demonstrate that the proposed scheme can attain an appropriate performance balance. In contrast, the MB-CWBAN scheme [15], the TA-CWBAN scheme [3], and the RP-CWBAN scheme [7] cannot offer such an attractive outcome under widely different CWBAN application load intensities. From the simulation analysis, the key observations of this study can be summarized: (i) by considering the anonymity of each WBAN, the GGBS is the best solution to solve the communication resource allocation problem; (ii) based on the tradeoff between egalitarian and utilitarian characteristics, the CBS can provide a fair-efficient solution for the computation resource allocation problem; (iii) according to the synergy of combining the GGBS and CBS, we can fully exploit the limited CWBAN system resources; and (iv) for real-world CWBAN operations, our step-by-step interactive approach can strike an appropriate performance balance in an online manner.

\section{Summary and Conclusions}

Cloudlet is an important technology that provides the performance improvement to many mobile applications. In particular, the seamless integration of cloudlet and WBANs provides tremendous opportunities for future network systems. However, so far, very little attention has ever been paid to cloudlet placement in WMANs, and there are few research papers about cloud-assisted WBANs. In this study, we propose a dual-phase bargaining game model to address the CWBAN resource allocation problem. For the communication resource allocation, the main idea of GGBS is adopted, and the CBS approach is applied for the computation resource allocation. These two bargaining processes are executed sequentially to tune the CWBAN resources while mixing the egalitarian and utilitarian principles. In order to evaluate the efficiency of the proposed solution, simulation is performed by measuring the payoff ratio, resource utilization, and fairness of CWBAN system. The simulation results demonstrate that the proposed scheme is very promising. Evidently, the proposed scheme leads to a well-balanced WBAN system performance, while other existing schemes cannot offer such an attractive system performance.

In the future, we plan to extend this work by incorporating machine learning characteristics with necessary modifications in the bargaining procedure. Additionally, we envision to implement a noncooperative approach based on the Bayesian game theory to achieve a collective control decision. Other possible future researches can include the dynamic behavior of WBANs in a critical emergency situation and the dynamic cache optimization for handling such emergency situations. Further, data security issue will be an interesting topic of the next steps of the future work.

\section{Data Availability}

The data used to support the findings of this study are available from the corresponding author upon request.

\section{Conflicts of Interest}

The author declares that there are no conflicts of interest regarding the publication of this paper.

\section{Authors' Contributions}

The author solely participated in the design of the study and performed the statistical analysis.

\section{Acknowledgments}

This research was supported by the MSIT (Ministry of Science and ICT), Korea, under the ITRC (Information Technology Research Center) support program (IITP-20192018-0-01799) supervised by the IITP (Institute for Information and communications Technology Planning and Evaluation) and was also supported by Basic Science Research Program through the National Research Foundation of Korea (NRF) funded by the Ministry of Education (NRF2018R1D1A1A09081759).

\section{References}

[1] Z. Liu, B. Liu, and C. W. Chen, "Joint power-rate-slot resource allocation in energy harvesting-powered wireless body area networks," IEEE Transactions on Vehicular Technology, vol. 67, no. 12, pp. 12152-12164, 2018.

[2] S. Sodagari, B. Bozorgchami, and H. Aghvami, "Technologies and challenges for cognitive radio enabled medical wireless body area networks," IEEE Access, vol. 6, pp. 29567-29586, 2018.

[3] S. Misra and A. Samanta, "Traffic-aware efficient mapping of wireless body area networks to health cloud service providers in critical emergency situations," IEEE Transactions on Mobile Computing, vol. 17, no. 12, pp. 2968-2981, 2018.

[4] D. He, S. Zeadally, and L. Wu, "Certificateless public auditing scheme for cloud-assisted wireless body area networks," IEEE Systems Journal, vol. 12, no. 1, pp. 64-73, 2018. 
[5] M. Quwaider and Y. Jararweh, "An efficient big data collection in body area networks," in Proceedings of the IEEE International Conference on Information and Communication Systems, pp. 1-6, Irbid, Jordan, April 2014.

[6] J. Wan, C. Zou, S. Ullah, C.-F. Lai, M. Zhou, and X. Wang, "Cloud-enabled wireless body area networks for pervasive healthcare," IEEE Network, vol. 27, no. 5, pp. 56-61, 2013.

[7] M. M. Hassan, H. Albakr, H. Al-Dossari, and A. Mohamed, "Resource provisioning for cloud-assisted body area network in a smart home environment," IEEE Access, vol. 5, pp. 13213-13224, 2017.

[8] H. Cao, V. Leung, C. Chow, and H. Chan, "Enabling technologies for wireless body area networks: a survey and outlook," IEEE Communications Magazine, vol. 47, no. 12, pp. 84-93, 2009.

[9] T. Gao, T. Massey, L. Selavo et al., "The advanced health and disaster Aid network: a light-weight wireless medical system for triage," IEEE Transactions on Biomedical Circuits and Systems, vol. 1, no. 3, pp. 203-216, 2007.

[10] A. Milenković, C. Otto, and EmilJovanov, "Wireless sensor networks for personal health monitoring: issues and an implementation," Computer Communications, vol. 29, no. 1314, pp. 2521-2533, 2006.

[11] S. Kim, Game Theory Applications in Network Design, IGI Global, Hershey, PA, U.S.A, 2014.

[12] M. A. Hinojosa, A. M. Mármol, and J. M. Zarzuelo, "Inequality averse multi-utilitarian bargaining solutions," International Journal of Game Theory, vol. 37, no. 4, pp. 597-618, 2008.

[13] M. Quwaider and Y. Jararweh, "A cloud supported model for efficient community health awareness," Pervasive and Mobile Computing, vol. 28, pp. 35-50, 2016.

[14] M. Quwaider and Y. Jararweh, "Cloudlet-based efficient data collection in wireless body area networks," Simulation Modelling Practice and Theory, vol. 50, pp. 57-71, 2015.

[15] S. Moulik, S. Misra, and A. Gaurav, "Cost-effective mapping between wireless body area networks and cloud service providers based on multi-stage bargaining," IEEE Transactions on Mobile Computing, vol. 16, no. 6, pp. 1573-1586, 2017.

[16] M. A. Hinojosa, A. M. Mármol, and J. M. Zarzuelo, MultiUtilitarian Bargaining Solutions, Universidad Pablo de Olavide, Department of Economics, Sevilla, Spain, 2007.

[17] C. Blackorby, W. Bossert, and D. Donaldson, "Generalized ginis and cooperative bargaining solutions," Econometrica, vol. 62, no. 5, pp. 1161-1178, 1994.

[18] A. Efe, "Oka and lin zhou, "the Choquet bargaining solutions," Games and Economic Behavior, vol. 33, no. 2, pp. 249-264, 2000.

[19] M. Dianati, X. Shen, and S. Naik, "A new fairness index for radio resource allocation in wireless networks," in Proceedings of the IEEE Wireless Communications And Networking Conference, vol. 2, pp. 712-715, New Orleans, LA, USA, March 2005. 